\title{
Comparison between different decorrelation techniques in vital sign detection
}

\author{
Angela Dell'Aversano*, Andrea Natale and Raffaele Solimene \\ Dipartimento di Ingegneria Industriale e dell'Informazione, \\ Second University of Naples, Aversa, Italy \\ *corresponding author, E-mail: angela.dellaversano@unina2. it
}

\begin{abstract}
The problem of detecting the breath activities of a human subject is addressed. A CW signal is used to probe the scene and the MUSIC algorithm is exploited to detect frequency doppler modulation introduced by chest movements. For this particular measurement configuration, the correlation matrix results rank deficient. In order to restore the rank, two decorrelation techniques are compared by exploiting numerical data.
\end{abstract}

\section{Introduction}

The contactless heartbeat and respiration detection of a human subject via radar sensing is a problem that is relevant in medical as well as security contexts (e.g. for apnea syndrome and baby monitor)[1] or for detecting human beings trapped under snow or debris after avalanche or quake events [2].

The underlying principle upon which those methods are based is the Doppler shift. More in detail, once a human body is exposed to continuous-wave (CW) microwave fields, the arising reflected signals turn out to be modulated in frequency (or equivalently in phase) due to body movements. Now, if the subject is at rest for some reasons, the only relevant movement is the one due to chest displacement owing to the breathing and heart beating. These movements are essentially nearly periodic and hence can be detected starting from the reflected field by adopting a suitable demodulation/filtering scheme.

Vital signs' detection is a complex task due to many impairing factors. Leakage between the TX and RX channels, null point problem, stability of local oscillator and strong clutter coming from environment are among the problems to be faced [3].

Most of breath frequency estimation approaches are based on Fourier transform [4] algorithms which are often combined with proper demodulation schemes in order to avoid some of the drawbacks mentioned above [5].

In this paper, in order to detect the Doppler shift, a MUSIC [6] based algorithm is adopted. The key idea is to reach a particular spectral decomposition of the data correlation matrix into the so-called signal and noise subspaces. In particular, the noise subspace is employed to build the pseudospectrum which "peaks", in the unknown space, just in correspondence of the looked parameters.

Here, the MUSIC algorithm is properly tailored to take into account that for the case at hand the received signal is

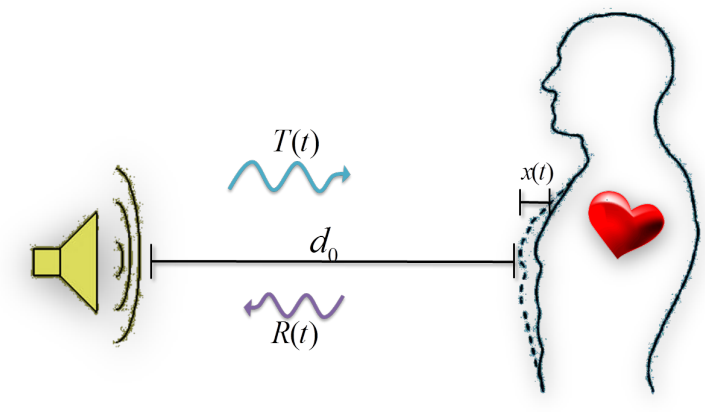

Figure 1: Scattering scenario: a human being is probed by a $\mathrm{CW}$ radio signal.

not a linear combination of complex exponentials. Moreover, as the signals representing the vital signs are correlated, a decorrelation stage has to be run before detection. Otherwise, the achievable performance rapidly decays so to cause missing detection.

In order to restore the rank of the correlation matrix, two decorrelation methods are compared: the spatial smoothing [7]-[8] and the Toeplitz based method [9].

Numerical examples are included in order to show how two methods work.

\section{Mathematic model}

Consider the scattering experiment schematically described in Figure 1. A human being is illuminated by the transmitted signal $T(t)$. Then the reflected modulated signal $R(t)$ arises and is collected in the receiving stage.

Neglecting amplitude variations, the transmitted signal $T(t)$ under $\mathrm{CW}$ regime can be expressed as

$$
T(t)=\cos (2 \pi f t+\phi(t))
$$

where $t$ is elapsed time, $f$ and $\phi(t)$ are the frequency and the phase noise of the local oscillator, respectively.

This signal impinges on a human target located at a nominal distance $d_{0}$. The chest introduces a time-varying displacement denote by $x(t)$. Accordingly, after the transmitted signal propagates back and forth for a total distance (between the transmitter and the receiver) equal to $2 d(t)=2 d_{0}+2 x(t)$, the received signal is collected; its 
expression is given by

$$
R(t) \approx \cos \left[2 \pi f t-\frac{4 \pi\left(d_{0}+x(t)\right)}{\lambda}+\phi\left(t-\frac{2 d_{0}}{c}\right)\right]
$$

where $c$ is the propagation velocity (the speed of light) and $\lambda$ is the wavelength in air, which equals $c / f$.

Chest movements are of course embedded in the phase term related to $x(t)$. Therefore, this is the factor that has to be single out in order to detect (if any) and possibly characterize the breath activities. Accordingly, the received signal is processed by the receiving channel and a MUSIC-based algorithm as depicted in Figure 2.

Note that a quadrature demodulation scheme is exploited in order to avoid the null point problem. Hence, the signal to be processed is given by

$$
B(t)=B_{I}(t)+\jmath B_{Q}(t)=\exp \left(\jmath \frac{4 \pi x(t)}{\lambda}+\jmath \theta(t)\right)
$$

where $\theta(t)=\frac{4 \pi d_{0}}{\lambda}+\Delta \phi(t)$ and $\Delta \phi(t)$ is the residual local oscillator phase noise, which usually can be neglected [10].

As the spatial displacement $x(t)$ is very small, it results that

$$
B(t) \approx \exp (\jmath \theta)\left(1+j \frac{4 \pi x(t)}{\lambda}\right)
$$

Actually, besides the signal in Equation (3), clutter contributions are always present, specially when there is an obstacle (i.e., a wall, etc.) between the person and the antenna system.

Therefore, the actual demodulated signal is

$$
B(t)=C+\exp (\jmath \theta)\left(1+j \frac{4 \pi x(t)}{\lambda}\right)
$$

where $C$ stays for the clutter and is not function of time $t$.

Note that the clutter introduces a static contribution (after demodulation) that can be eliminated by filtering out the continuous component. This filtered signal is denoted as

$$
\tilde{B}(t)=\exp (\jmath \theta) j \frac{4 \pi x(t)}{\lambda}
$$

and represents the signal that is actually passed to the MUSIC stage.

From the theory of Fourier series, any time-varying periodic displacement $x(t)$ can be viewed as the combination of a series of single-tone signals. Therefore, for the ease of analysis and without loss of generality, it is assumed that the chest movement $x(t)$ is described as the sum of $x_{h}(t)=A_{h} \cos \left(2 \pi f_{h} t\right)$, due to heartbeat, and $x_{r}(t)=A_{r} \cos \left(2 \pi f_{r} t\right)$ due to respiration.

\section{MUltiple SIgnal Classification (MUSIC)}

The MUSIC algorithm is a powerful method to estimate frequencies embedded in a signal.

The standard formulation applies to a linear combination of complex exponentials. Therefore, here, it is need to adapt this method to the problem at hand.

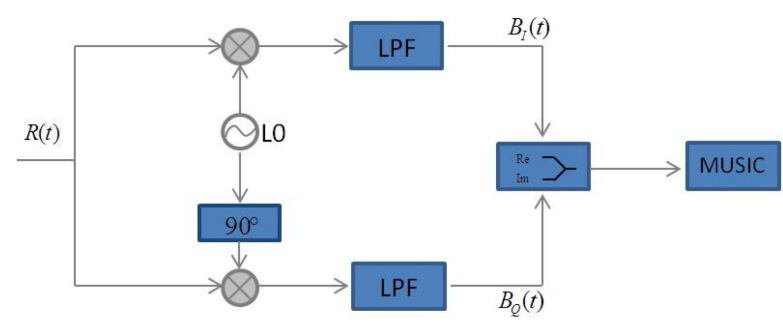

Figure 2: Schematic of the receiving stage.

Assume that the reflected signal is collected in correspondence of $L$ instants of time $t_{n}$ so that $t_{n+1}-t_{n}=\Delta t$ is the sampling interval. Say $\tilde{\mathbf{b}}$ the data column vector corresponding to the time vector $\mathbf{t}$. Then, Equation (5) can be recast in a matrix form as

$$
\tilde{\mathbf{b}}=\mathbf{C a}=\left[\cos \left(2 \pi \mathbf{t} f_{1}\right) \ldots \cos \left(2 \pi \mathbf{t} f_{d}\right)\right] \mathbf{a}
$$

where $d$ is the number of harmonics and a the coefficient column $a_{i}=\exp (\jmath \theta) j \frac{4 \pi A_{i}}{\lambda}$ (in our case $d=2$ and $A_{1}=$ $A_{h}$ and $A_{2}=A_{r}$ ).

In order to build the pseudospectrum, the data space is factorized in the so-called signal and noise subspaces. To this end, it is noted that the matrix $\mathbf{C}$ can be expressed as $\mathbf{C}=\mathbf{E}^{-}+\mathbf{E}^{+}$where $\mathbf{E}^{-}=\left[\exp \left(-j 2 \pi \mathbf{t} f_{1}\right) \ldots \exp \left(-j 2 \pi \mathbf{t} f_{d}\right)\right]$ and $\mathbf{E}^{+}=$ $\left[\exp \left(j 2 \pi \mathbf{t} f_{1}\right) \ldots \exp \left(j 2 \pi \mathbf{t} f_{d}\right)\right]$.

Accordingly, the signal subspace corresponds to that range of $\mathbf{C}$, i.e., $\mathcal{R}(\mathbf{C})$, which in turn $\mathcal{R}(\mathbf{C})=\mathcal{R}\left(\mathbf{E}^{-}\right) \cup \mathcal{R}\left(\mathbf{E}^{+}\right)$. Therefore, $\mathcal{R}(\mathbf{C})=$ $\operatorname{span}\left\{\exp \left(-j 2 \pi \mathbf{t} f_{1}\right), \ldots, \exp \left(-j 2 \pi \mathbf{t} f_{d}\right), \exp \left(j 2 \pi \mathbf{t} f_{1}\right)\right.$, $\left.\ldots, \exp \left(j 2 \pi \mathbf{t} f_{d}\right)\right\}$. The noise subspace is orthogonal to both $\mathcal{R}\left(\mathbf{E}^{-}\right)$and $\mathcal{R}\left(\mathbf{E}^{+}\right)$. Hence, also for the case at hand, the pseudospectrum can be achieved by considering as steering vector only exponential like vectors (as in usual MUSIC).

Formally, the pseudospectrum is as follows

$$
\Phi(f)=\frac{1}{\|\mathcal{P}[\exp (j 2 \pi f \mathbf{t})]\|^{2}}
$$

where $\mathcal{P}$ is the projection operator onto the noise subspace which depends on the adopted decorrelation algorithm.

\section{Decorrelation methods}

For the particular scenario addressed herein, it is obvious that the correlation matrix corresponding to (6) is rank deficient. So the rank need to be restored by some decorrelation techniques. In this section two different algorithms are described.

\subsection{Spatial Smoothing}

In Spatial Smoothing technique (SS) the data, consisting of $L$ time samples, is separated in $M_{d}=L-N+1$ overlapped sub-arrays composed by $N$ samples, so that $\tilde{\mathbf{b}}_{\mathbf{k}}=\tilde{B}\left(t_{k}, \ldots, t_{k+N}\right)$, with $k \in\left[1,2, \ldots, M_{d}\right], M_{d}$ 


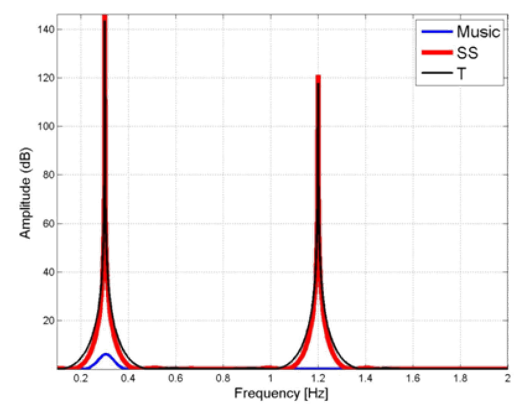

(a)

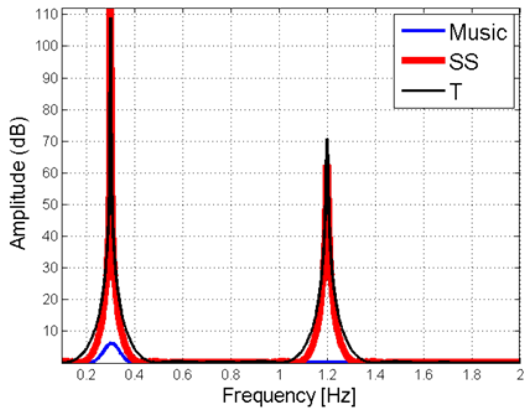

(d)

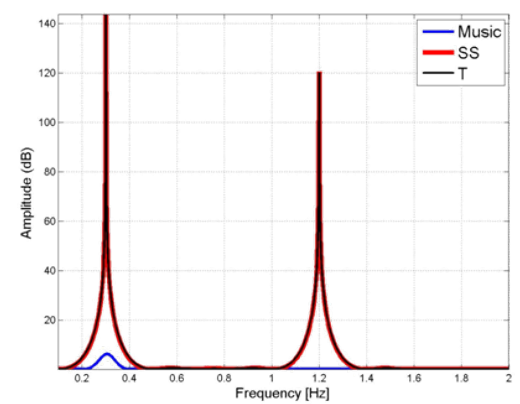

(b)

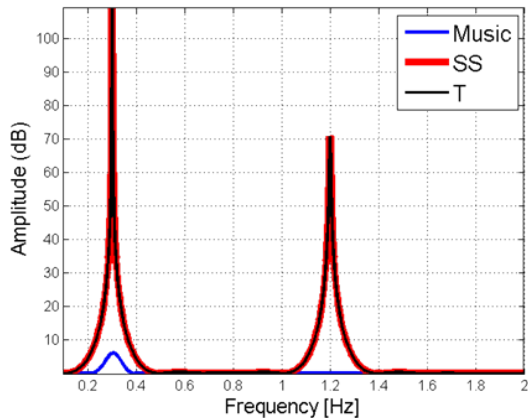

(e)

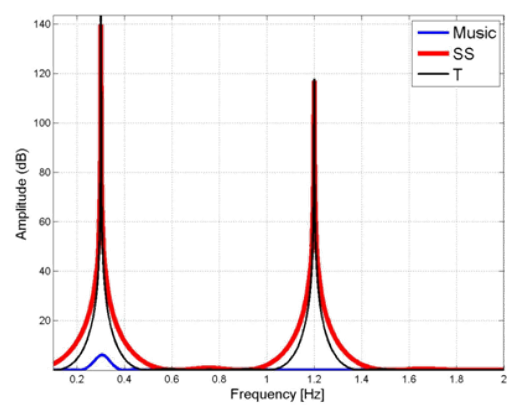

(c)

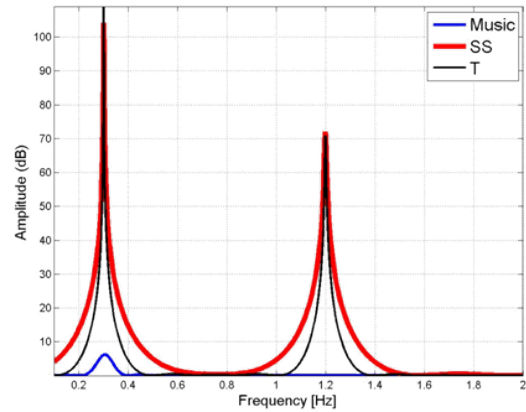

(f)

Figure 3: Synthetic data with acquisition time equal to 10s. Pseudospectrum comparison between SS (red line) and Toeplitz (black line) methods by varying the number of samples and the decorrelation index: (a) $L=270, M_{d}=53$, (b) $L=270$, $M_{d}=L / 2$, (c) $L=270, M_{d}=153$, (d) $L=80, M_{d}=21$, (e) $L=80, M_{d}=L / 2$, (f) $L=80, M_{d}=61$

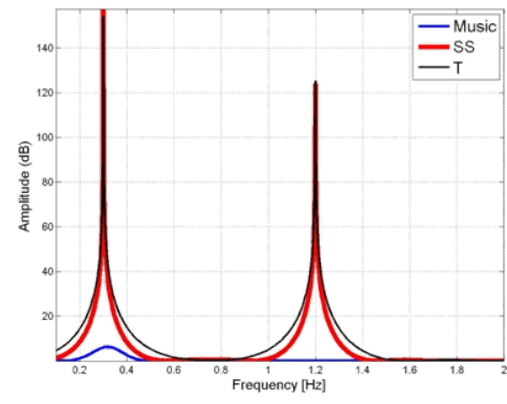

(a)

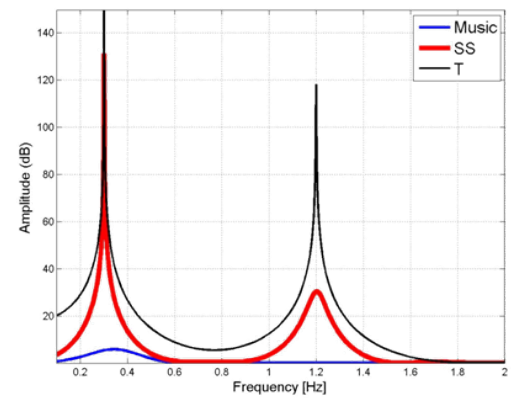

(d)

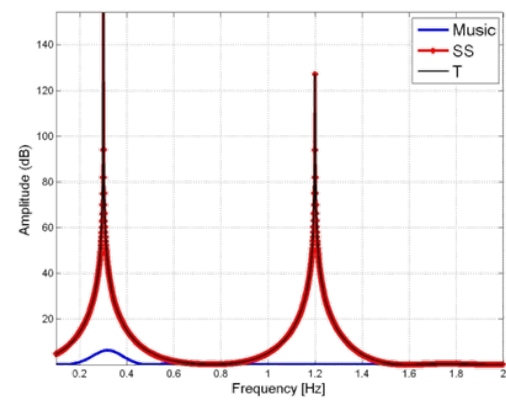

(b)

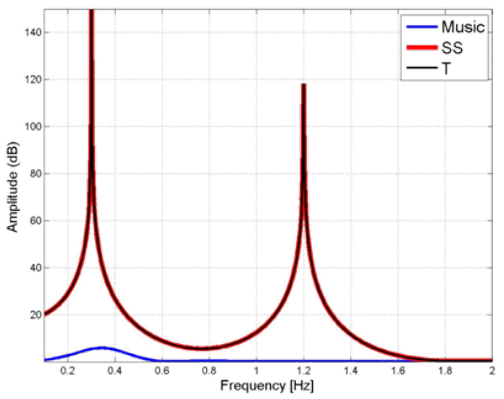

(e)

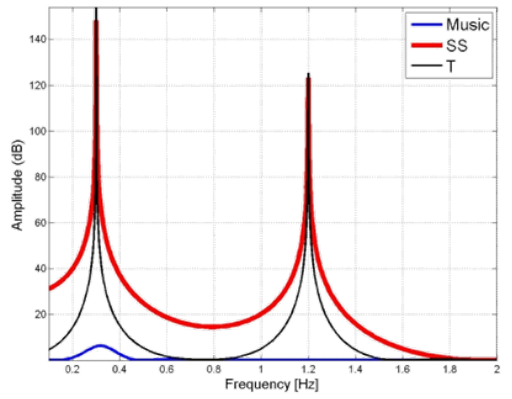

(c)

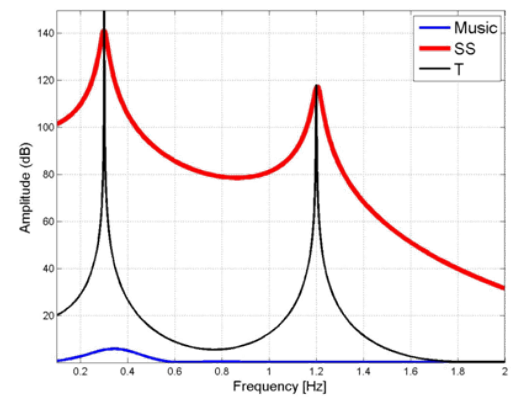

(f)

Figure 4: Synthetic data by varying the acquisition time and the decorrelation index. Pseudospectrum comparison between SS (red line) and Toeplitz (black line): (a) $\Delta t L=5 s, M_{d}=51$, (b) $\Delta t L=5 s, M_{d}=L / 2=100$, (c) $\Delta t L=5 s, M_{d}=151$, (d) $\Delta t L=3 s, M_{d}=11$, (e) $\Delta t L=3 s, M_{d}=L / 2=60$, (f) $\Delta t L=3 s, M_{d}=101$ 
being the decorrelation index. Accordingly, the correlation matrix is estimated as

$$
S_{S S}=\frac{1}{M_{d}} \sum_{k=1}^{M_{d}} \mathbf{r}_{k}
$$

where

$$
\mathbf{r}_{k}=\tilde{\mathbf{b}}_{k} \tilde{\mathbf{b}}_{k}^{H}=\mathbf{D} \mathbf{H}_{k} \mathbf{D}^{H}
$$

is the correlation matrix calculated for the $k$-th sub-array, $\mathbf{D}=\left[\cos \left(2 \pi \mathbf{t}_{i} f_{h}\right), \cos \left(2 \pi \mathbf{t} f_{r}\right), \sin \left(2 \pi \mathbf{t} f_{h}\right)\right.$,

$\left.\sin \left(2 \pi \mathbf{t} f_{r}\right)\right]$ and $(\cdot)^{H}$ denotes the complex conjugate transpose. $\mathbf{H}_{k}$ is a $4 \times 4$ matrix. If the smoothing is properly achieved then the rank of $\sum_{k=1}^{M_{d}} \mathbf{H}_{k}$ should be 4 .

In order to identify the signal and noise subspace, the Singular Value Decomposition (SVD) is applied to $S_{S S}$. By checking singular values, the noise subspace is defined as the set of singular values that get small with respect to the first one. In particular, note that for the case at hand, due to particular form of the matrix $\mathbf{C}$, the number of the relevant singular values (i.e., the signal subspace dimension) is twice as the number of frequencies. Finally, the projection operator $\mathcal{P}$ picks the corresponding left singular function of $S_{S S}$ in order to build the pseudospectrum. Note that after the smoothing, data is as it were collected only over the instants (7), with $\mathbf{t}=\mathbf{t}(1, \ldots, N)$.

\subsection{Toeplitz based algorithm}

This method is based on building up Toeplitz matrices for each row of the correlation matrix $\mathbf{r}=\tilde{\mathbf{b}} \tilde{\mathbf{b}}^{H}$ [9]. Its introduction is motivated by the need to avoid reducing the time interval used while constructing the pseudospectrum that occurs in the Spatial Smoothing procedure.

The $l$-th Toeplitz matrix is defined as

$$
\begin{gathered}
\mathbf{R}_{l}= \\
{\left[\begin{array}{llll}
\mathbf{r}\left(l, \frac{L}{2}\right) & \mathbf{r}\left(l, \frac{L}{2}+1\right) & \cdots & \mathbf{r}(l, L-1) \\
\mathbf{r}\left(l, \frac{L}{2}-1\right) & \mathbf{r}\left(l, \frac{L}{2}\right) & \cdots & \mathbf{r}(l, L-2) \\
\vdots & \cdots & \ddots & \vdots \\
\mathbf{r}(l, 1) & \mathbf{r}(l, 2) & \cdots & \mathbf{r}\left(l, \frac{L}{2}\right)
\end{array}\right]=} \\
\\
\quad=\mathbf{G S}_{l} \mathbf{G}^{H}
\end{gathered}
$$

where $\mathbf{G}=\left[\begin{array}{llll}\mathbf{g}\left(f_{r}\right) & \mathbf{g}^{*}\left(f_{r}\right) & \mathbf{g}\left(f_{h}\right) & \mathbf{g}^{*}\left(f_{h}\right)\end{array}\right]$ is the steering matrix where $\mathbf{g}\left(f_{d}\right)=$ $\left[\exp \left(j 2 \pi t_{L / 2} f_{d}\right), \exp \left(j 2 \pi t_{L / 2-1} f_{d}\right), \cdots, \exp \left(j 2 \pi t_{1} f_{d}\right)\right]^{T}$ $(d=r, h)$ and $\mathbf{S}_{l}$ is $4 \times 4$ diagonal matrix that can be shown to be full rank.

Then the SVD factorization is applied to the following matrix

$$
S_{T}=\sum_{l=1}^{L / 2} \mathbf{R}_{l}^{H} \mathbf{R}_{l}
$$

with $\mathbf{t}=\mathbf{t}(1, \cdots, L / 2)$ in (7).

It is noted that also this method entails a reduction of the time interval. But in this case only half the time interval is lost to achieve decorrelation.

It is interesting to compare both methods. This is the main aim of this paper and will be achieved numerically in the next section. However, we find interesting to show some analytical arguments we found that establish a clear link between them. More in detail, comparing the $i, j$-th entry of SS correlation matrix

$$
\left\{S_{S S}\right\}_{i j}=\frac{1}{M_{d}} \sum_{k=1}^{M_{d}} b_{i+k-1} b_{j+k-1}^{*}
$$

and that of Toeplitz correlation matrix

$$
\left\{S_{T}\right\}_{i j}=F \sum_{k=1}^{L / 2} b_{i+k-1} b_{j+k-1}^{*}
$$

with $F=\sum_{k=1}^{L / 2}\left|b_{k}\right|^{2}$, it is easy to note that, unless unessential constant, the entries of both matrices are the same when $M_{d}=L / 2$. This means that for $M_{d}=L / 2$ the two methods are practically the same. Also, this solves the problem connected to the choice of $M_{d}$ in the SS algorithm. Indeed, by choosing $M_{d}=L / 2$ one is sure that SS fully recovers the rank.

\section{Numerical Results}

In this section the analysis is conducted with numerical data. The comparison between the two decorrelation methods is carried out in terms of dynamic range and resolution and with respect to the signal to noise ratio (SNR), number of time samples and time interval duration.

The demodulated signal taken into account is

$$
\tilde{\mathbf{b}}=A_{r} \cos \left(2 \pi f_{r} \mathbf{t}\right)+A_{h} \cos \left(2 \pi f_{h} \mathbf{t}\right)+n(\mathbf{t})
$$

with $A_{r}=1.5 \mathrm{~mm}, A_{h}=0.001 \mathrm{~mm}, f_{r}=0.3 \mathrm{~Hz}$ and $f_{h}=1.2 \mathrm{~Hz}, n(\mathbf{t})$ denotes the noise which is assumed to be AWGN.

In all the following examples, the pseudospectra are plotted in log-scale: the blue line indicates the MUSIC pseudospectrum without decorrelation step, instead, red and black lines show the pseudospectra with SS and Toeplitz decorrelation methods, respectively.

The reflected signal is collected at most over $L=400$ samples for an interval of time of $10 \mathrm{~s}$.

As first example, noiseless data are considered while the number of samples is reduced at 270 (Figure 3(a)-(c)) and then at 80 (Figure 3(d)-(e)). The analysis is conducted by varying the correlation index $M_{d}$ in the SS method (red line) in order to appreciate the differences with respect to Toeplitz technique (black line).

As can be noted, in panel (b) and (e), when $M_{d}$ is chosen equal to $L / 2$, the performance are the same in perfect agreement with the discussion reported above. On the other 


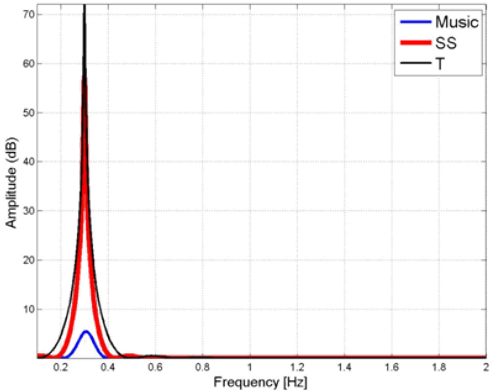

(a)

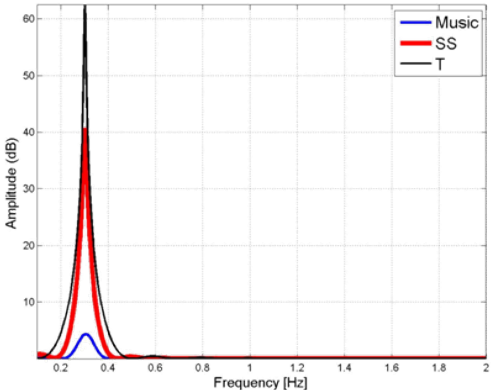

(d)

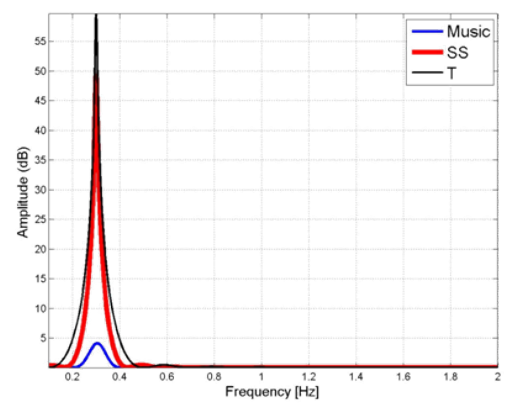

(g)

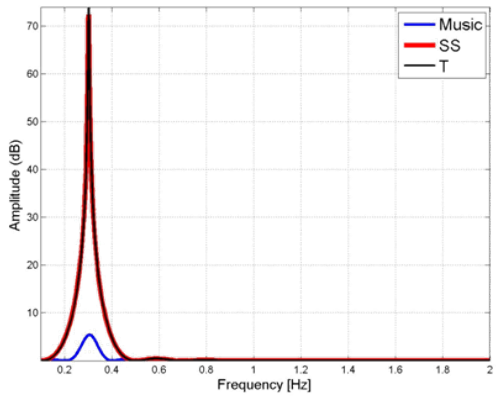

(b)

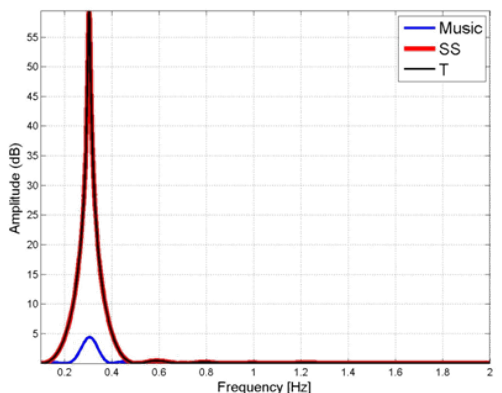

(e)

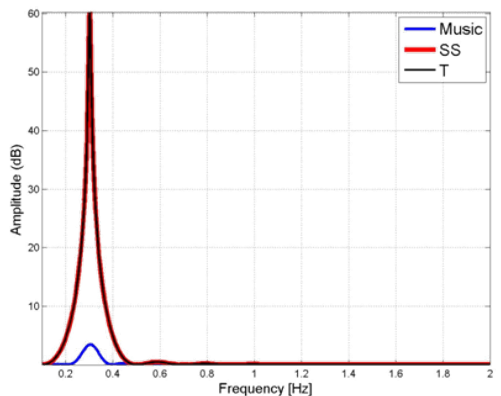

(h)

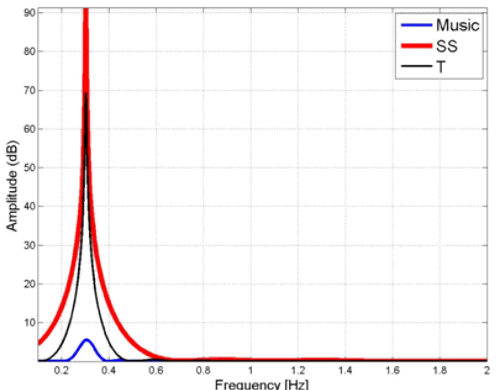

(c)

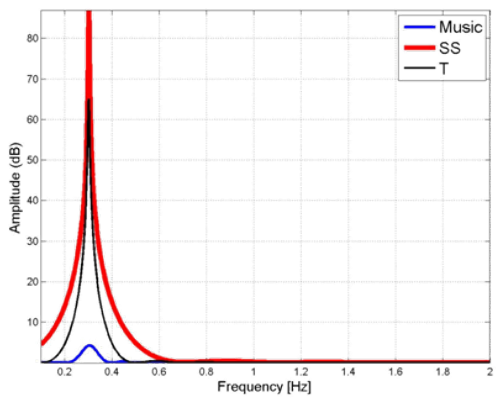

(f)

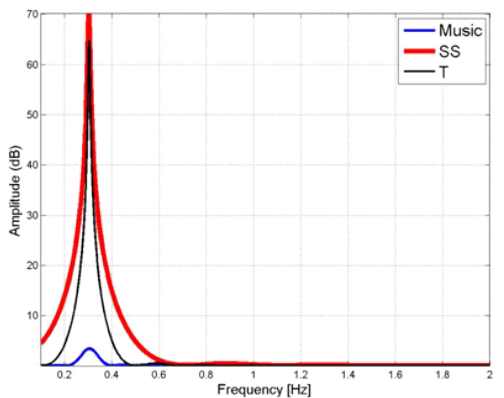

(i)

Figure 5: Synthetic data with $L \Delta t=10 s$ and $L=400$. Comparing SS (red line) and Toeplitz (black line) methods by varying the SNR level and the correlation index: (a) $S N R=20 \mathrm{~dB}, M_{d}=101$, (b) $S N R=20 \mathrm{~dB}, M_{d}=L / 2$, (c) $S N R=20 \mathrm{~dB}$, $M_{d}=301$, (d) $S N R=10 d B, M_{d}=101$, (e) $S N R=10 d B, M_{d}=L / 2$, (f) $S N R=10 d B, M_{d}=301$, (g) $S N R=5 d B$, $M_{d}=101$, (h) $S N R=5 d B, M_{d}=L / 2$, (i) $S N R=5 d B, M_{d}=301$ 
hand, when $M_{d}=53<L / 2$ (panel (a), (d)) the SS decorrelation performs better than the Toeplitz method in terms of resolution, whereas when $M_{d}=153>L / 2$ (panel (c), (f)) the opposite is true. This clearly occurs because $M_{d}$ actually dictates the effective length of the time interval involved in the pseudospectrum formation.

In Figure 4 the role of time interval is studied keeping fixed the sampling step. In detail, in panels (a)-(c) the acquisition time is reduced to 5 seconds $(L=200)$ whereas in panels (d)-(f) to 3 seconds ( $L=120)$. Of course, as the overall acquisition time has been reduced the pseudospectra tend to enlarge. Furthermore, the same trade as above as far as $M_{d}$ is concerned is practically observed. However, now, when $M_{d}<L / 2$ (here $M_{d}=11$, see Figure 4(d)) the SS also exhibits a loss in the dynamic range. This is because in this case the smoothing procedure does not work perfectly. Whereas, when $M_{d}>L / 2\left(M_{d}=101\right.$, see Figure 4(f)), the SS shows a dramatic loss of resolution.

Finally, we turn to address the effect of noise on data.

In Figure 5 the results retuned by the two methods at hand are displayed by varying the Signal to Noise Ratio (SNR). In particular, in the panels (a)-(c) $S N R$ is equal to $20 d B$, in (d)-(f) the $S N R=10 d B$ and in (g)-(i) $S N R=$ $5 d B$.

As expected, the presence of noise does not allow to detect the heartbeat harmonic due to its very low amplitude $A_{h}$. Also, both the methods appears relatively stable against noise, even though the dynamic range reduces as compared to the noiseless case. Finally, it is noted that whether $M_{d}<L / 2$ or $M_{d}>L / 2$ the SS returns lower or higher (than the Toeplitz method) pseudospectra. This can be explained that when $M_{d}>L / 2$ Equation (8) entails a more "strong" averaging that tends to reduce the noise. However, the cost to pay is a loss of resolution.

\section{Conclusions}

In this paper, two different decorrelation methods for vital sign detection have been compared. In particular, the analysis has been conducted in terms of dynamic range and resolution for Spatial Smoothing and Toeplitz methods.

It has been demonstrated that the SS and Toeplitz techniques show the same performance with a suitable choice of decorrelation index, in particular when it is equal to half of number of the available samples $(L / 2)$.

The performance of SS method depends by the choice of decorrelation index. Indeed, the results have shown that when $M_{d}<L / 2$ the pseudospetrum have a loss of dynamic range and an increasing of resolution and for $M_{d}>L / 2$ the opposite holds true.

\section{References}

[1] A.-D. Droitcour, V.-M. Lubecke, J. Lin and O. BoricLubecke, A microwave radio for Doppler radar sensing of vital signs, Microwave Symposium Digest, 2001 IEEE MTT-S International 1:175-178, 2001.
[2] K. M. Chen, J. Zhang and A. Norman, Microwave life-detection systems for searching human subjects under earthquake rubble and behind barrier, IEEE Transactions on Biomedical Engineering 47: 105$114,2000$.

[3] C. Li, V. M. Lubecke, O. Boric-Lubecke, J. Lin, A Review on Recent Advances in Doppler Radar Sensors for Noncontact Healthcare Monitoring, IEEE Transactions on Microwave Theory and Techniques 61:2046-2060, 2013.

[4] B. Lohman, O. Boric-Lubecke, V. M. Lubecke, P. W. Ong and M. M. Sondhi, A digital signal processor for Doppler radar sensing of vital signs, IEEE Engineering in Medicine and Biology 21: 161-164, 2002.

[5] C. Li, Y. Xiao and J. Lin, Experiment and spectral analysis of a lowpower Ka-band heartbeat detector measuring from four sides of a human body, IEEE Transactions on Microwave Theory and Techniques 54:4464-4471, 2006.

[6] R.O. Schmidt, Multiple emitter location and signal parameter estimation, IEEE Transactions on Antennas and Propagation 34:276-280, 1986.

[7] S. Tie-Jun, W. Mati, K. Thomas, On Spatial Smoothing for Direction-of-Arrival Estimation of Coherent Signals, IEEE Transaction on Acoustics, Speech and Signal Processing 33: 806-811, 1985.

[8] C. Qing, L. Ruonlun, On the Explanation of Spatial Smoothing in MUSIC Algorithm for Coherent Sources, International Conference on Information Science and Technology, Nanjing, China, pp.699-702, 2011.

[9] Q. Cheng, H. Lei, Z. Wen-Jun and S. Hing Cheung, Direction of Arrival Estimation for Coherent Signals Without Knowledge of Source Number, IEEE Sensors Journal 14:3267-3273, 2014.

[10] M. C. Budge, M. P. Burt, Range correlation effects on phase and amplitude noise,IEEE Southeastcon '93, Proceedings, Charlotte, NC, 1993. 